\section{Case Reports in Oncology}

\title{
Sparing Surgery for the Successful Treatment of Thyroid Papillary Carcinoma Invading the Trachea: A Case Report
}

\author{
Denis Kulbakin ${ }^{a, b}$ Timofey Chekalkin ${ }^{a, c}$ Marat Muhamedov \\ Evgeniy Choynzonov ${ }^{b}$ Ji-hoon Kang ${ }^{c}$ Seung-baik Kang ${ }^{d}$ \\ Victor Gunther ${ }^{\text {a }}$ \\ ${ }^{a}$ Tomsk State University, Tomsk, Russian Federation; ${ }^{b}$ Tomsk Cancer Research Institute, \\ Tomsk, Russian Federation; 'Kang \& Park Medical Co. Ltd., Cheongju, South Korea; \\ ${ }^{\mathrm{d}}$ Seoul National University Hospital, Seoul, South Korea
}

\section{Keywords}

Sparing surgery · Thyroid papillary carcinoma $\cdot$ Tracheal reconstruction · Prefabrication . Knitted TiNi-based mesh endograft

\begin{abstract}
Published reports on salvage treatment for trachea reconstruction after total thyroidectomy or partial tracheotomy are available, some of them using structures of the trachea itself, auricular cartilage, a musculocutaneous flap, or other methods. In our report, we emphasize the importance of a search for a new material and approach for sparing surgery. The purpose of this article is to describe a case of a successful sparing surgery in a patient with advanced thyroid papillary carcinoma invading the trachea. After total thyroidectomy in 2012, partial resection of the trachea was performed in 2014 . The lesion defect was $5.5 \times 2.3 \mathrm{~cm}$ in size, located between 4 (2nd-6th) tracheal cartilaginous rings and involving about a semicircumference. It was reconstructed with the aid of the knitted TiNi-based mesh endograft, which has been prefabricated in the sternocleidomastoid muscle and further covered with the skin draped over the wound. The tracheostoma was fully closed 6 weeks after the surgery. There
\end{abstract}




\section{Case Reports in Oncology}

Kulbakin et al.: Sparing Surgery for the Successful Treatment of Thyroid Papillary Carcinoma Invading the Trachea: A Case Report

were neither side effects nor complications. This kind of tracheal surgery for extensive lesions demonstrates good functional and cosmetic outcomes.

\section{Introduction}

Thyroid cancer is the 10th commonest cause of cancer in Russian women, and the agespecific incidence of thyroid cancer has been on the rise. While most thyroid cancers have a good prognosis, those that show tracheal invasion often show poor survival because of extensive resection or tumor recurrence. Surgical intervention is the key point of treatment, and appropriate surgical planning is essential to get a promising prognosis, postsurgical quality of life, and social rehabilitation of a patient [1].

Despite an increasing role of nonadjuvant radiotherapy and/or chemotherapy in the treatment of thyroid cancer, the surgical approach is still used when recurrence is treated after radiotherapy/chemoradiotherapy or in the case of combined treatment of patients with thyroid papillary carcinoma invading the trachea [1]. The extensive resection that may be necessary for locally invasive thyroid cancer has led some surgeons to attempt to conservatively approach these tumors using peeling or shaving techniques aiming at preserving function [2, 3]. Such conservative approaches rely on the administration of postoperative radioactive iodine with/without external beam radiotherapy to manage a microscopic disease. Many patients with locally advanced thyroid cancer tend to be older patients with more poorly differentiated histologic variants. Tumors in such patients can be nonradioactive iodine avid, and the response to radioactive iodine therapy can be disappointing [4].

Recently, the importance of sparing surgery has also increased in clinical head and neck oncology. It fully applies, for instance, to the treatment of patients with laryngeal cancer [5]. No doubt, a salvage treatment cannot be considered as a primary method. Reconstruction should not be the only objective of treatment. Better functional and cosmetic result for a patient should also be an objective [6].

In this report, we present a patient with advanced thyroid papillary carcinoma involving the trachea, who has previously undergone total thyroidectomy followed by radioactive iodine therapy in 2012. Tumor recurrence was verified in 2013. Further, we successfully treated the patient by double-stage surgery in 2014. The first one was a window resection of the trachea followed by an open tracheostomy, and the formed knitted TiNi-based mesh endograft (KTNME), as reported by Muhamedov et al. [5], was implanted subcutaneously in the sternocleidomastoid muscle for 5 weeks. The second stage was the reconstruction of the large tracheal defect with the harvested endograft and the skin draping over the wound.

\section{Case Presentation}

The patient presented here is a 54-year-old female who was referred and admitted to the hospital of the Tomsk Cancer Research Institute on April 2, 2014 (clinical case \#B273xx). The patient underwent total thyroidectomy for papillary thyroid cancer (type $\mathrm{T}_{4} \mathrm{~N}_{\mathrm{x}} \mathrm{M}_{0}$ ) in June 2012 when the cancer directly involved the tracheal wall, followed by radioactive iodine therapy in the Vladivostok Central Clinical Hospital in July 2012. Apparently, she was considered with no evidence of disease until December 2013 when the mass in her trachea that she noticed had been gradually increasing in size within the recent months, and the histological findings verified well-differentiated papillary carcinoma recurrence. 


\section{Case Reports in Oncology}

There were objectively no remarkable signs except for a neck incisional scar and the subcutaneous, rounded, newly grown palpatory mass detected (about $1.5 \mathrm{~cm}$ in diameter). She had other symptoms such as dyspnea at rest, hoarseness, and minor hemoptysis. Fiberoptic endoscopy and magnetic resonance imaging (MRI) revealed a circumferential stenosis on the right side below the vocal cord, invading the lumen of the trachea. A submucosal mass from the 2 nd to the 5 th tracheal ring with an irregular surface penetrating the mucosa with signs of ulceration and erosion was noted (Fig. 1).

According to the above findings, the case was classified as $\mathrm{T}_{4} \mathrm{~N}_{0} \mathrm{M}_{0}$, and a double-stage surgical treatment was scheduled (Fig. 2).

The first stage was performed on April 15, 2014. To involve the platysma muscle, the Ushaped incision was made alongside the postoperative scar (Fig. 3a). Subcutaneous fat was removed with electrocautery to aid in exposure and to prevent later fat necrosis. Dissection proceeded through the platysma until the midline raphe between the strap muscles was identified (Fig. 3b). The sternocleidomastoid muscle was thus separated, retracted laterally, and preserved. By tracing, the right recurrent laryngeal nerve was assessed to be intact and half of the trachea $(5.5 \times 2.3 \mathrm{~cm})$ was dissected including 5 cartilaginous rings together with the left recurrent laryngeal nerve. The tumor was removed together with the invaded paratracheal tissue bilaterally (Fig. 3c). The medial edges of the sternocleidomastoid muscle were sutured to a rectangular tracheal opening. To avoid tracheal stenosis, the anterior side of the defect was open, and a stent tube was kept in place. For muscle-flap prefabrication, the prepared KTNME, as described by Muhamedov et al. [5], was implanted into the sternocleidomastoid muscle (Fig. 3d). The cut edges of the tracheal opening were circumferentially overedged to the skin with a few absorbable sutures to facilitate cannulation. After the airway was confirmed intact based on $\mathrm{CO}_{2}$ return and bilateral breath sounds, the tracheostomy tube was secured to the skin with 4-0 sutures (Fig. 3e).

To avoid the risk of subcutaneous emphysema and subsequent pneumomediastinum, the skin was not tightly closed. A sponge soaked with iodine between the skin and the flange was placed for $24 \mathrm{~h}$ to deflect infection and anxiety about minor oozing of the skin edge. The patient was extubated after 7 days, and there was no granulation tissue or stenosis of the tracheal lumen 3 weeks thereafter (Fig. 4).

The reconstructive stage was performed on May 19, 2014. The O-shaped incision around the formed tracheotomy having the gap $1.5 \mathrm{~cm}$ from the right edge was made. A cellulocutaneous flap was separated and overturned so that the epidermis overlapped the tracheostomy lumen (Fig. 5a). This technique allowed the inner lining to be formed. The harvested KTNME after prefabrication was separated with the pedicle flap of the medial edge of the sternocleidomastoid muscle (Fig. 5b), placed over the trachea, and sutured to the trachea (Fig. 5c). The prefabricated KTNME was noted to be substantial and completely covered with the tissue. The remaining defect in the anterior part of trachea was closed without any special procedure. The sliding skin flap was swung over the wound followed by aesthetic wound closure to achieve optimal cosmetic results with minimal scarring (Fig. 5d).

The tracheal stoma was fully closed 6 weeks after the surgery. There were neither side effects nor complications. Postoperative bronchoscopy revealed a pink-coated part in the tracheal mucosa with no stenosis (Fig. 5e). The patient was discharged on June 3, 2014, and referred back home to be supervised by a local hospital, where she was socially rehabilitated in full. The patient remains alive and without any evidence of recurrence or complaints well over 24 months after surgery. She continued her job 2 months after discharge and calls us every 6 months to confirm her health status and current matters. 


\section{Discussion}

The possibility to perform a sparing surgery depends on several factors, including the availability of adequate material for the tracheal replacement after lesion [7, 8]. However, functional outcomes of such a kind of the treatment do not always satisfy both patients and clinicians given that a large portion of the trachea is to be resected in locally advanced thyroid papillary carcinoma invading the trachea. Some surgical methods, on account of the improvement, standardization, and more widespread use of reconstructive surgery with microvascular flaps, for the management of tracheal invasion by thyroid carcinoma have been reported in the literature, so far with no consensus on the best approach [3]. The development of techniques and the selection of an optimal material for the tracheal reconstruction remains the urgent aim of modern head and neck surgical oncology [9]. The brief overview of published articles on tracheal sparing surgery studies reflects, in fact, that there are no commercially produced implants for a wide clinical practice in cases of extensive lesions of the trachea. Since it is a mobile organ when breathing, swallowing, speaking, eating etc., it is critical to raise standards regarding the material selection for the replacement [10]. To succeed, clinicians should make their unconventional choice of what approach to use and how to perform the correct reconstruction.

As a rule, the structures involved in cases of invasive thyroid papillary carcinoma are mostly the trachea, larynx, laryngeal nerve, and other surrounding sites. Since a regional spread, increased incidence of local recurrence and distant metastasis are common ways of invasion to the surrounding structures, it is crucial to control local invasion, which makes treatment relatively difficult. The management of locally invasive, well-differentiated papillary carcinoma of the thyroid is disputable. Palliative surgery had been the most common surgical procedure used for the treatment of patients with thyroid carcinoma invading the trachea for many years. However, the shaving-off procedure was reported to be often inadequate, and the postoperative survival rate was much worse in patients undergoing partial resection than in those undergoing total resection [11]. Hence, total resection is always desired to be attempted when possible.

For adequate surgery, as in our case, accurate preoperative examination of the tracheal invasion including bronchoscopy, CT, and MRI is required to clarify the involvement of adjacent organs.

The generally accepted technique for the reconstruction of circular tracheal lesions is to form the end-to-end primary anastomosis between the proximal and distal margins of the defect. However, this is feasible for small (less than 3 rings) circular defects [12], and a larger area of invasion may limit the feasibility of partial tracheal resection because it may lead to kinking or stenosis of the trachea after repair. Contraindications include extensive involvement of the trachea, such that 6 or more tracheal rings need to be resected or simultaneous invasion of the esophagus occurs. Moreover, in cancer patients, the upward trend in postoperative complications is probable, including a failure of the anastomosis or scar stenosis due to a previous treatment and the weak reparative ability of the tissue.

In case of extensive tracheal defects (more than $5 \mathrm{~cm}$ ), the most reliable method is reconstructive surgery, which involves a layerwise restoration of the tracheal wall. Such an approach assumes a fusion of the framework and a thin flap, which adequately replaces the frame function of the trachea without narrowing the lumen. Currently, none of the known flaps meets the above-mentioned requirements. Therefore, the suggested KTNME is promising for tracheal reconstruction. There are several similar studies that aimed at exploring tracheal reconstruction considering the frame function, but they are strictly experimental 


\section{Case Reports in Oncology}

and still not applicable in cases of large defects of the trachea [13]. It should be pointed out that applying the implant in large defects of the trachea has to be done without any contact with the lumen when there are signs of a primary inflammation. It is suggested to ensure strict adherence to the criteria, otherwise the inflammatory process that occurs in situ eventually affects the consistency of the graft and leads to granulation tissue growth and a failure of the whole reconstruction stage [14].

In summary, in order to succeed in a complex sparing surgery, the obvious procedure is to use the prefabricated endograft covered with a thin-walled lining by the adjacent tissues, as we did. This technique implies 2 stages of reconstruction, which do not contravene the modern concept of sparing surgery as repeated corrective treatments to restore the lumen are necessary [7]. A two-stage surgical treatment has been applied before in cases of large tracheal lesion defects: first, after resection of the tumor, the tracheal wall and skin are sutured for the development of a persistent tracheocutaneous fistula "trough". Second, delayed closure of the tracheal defect was performed [15]. Unfortunately, compared to our method, this technique involves long-term treatment and is also not always aesthetically satisfactory because of the need for harvesting a local flap.

\section{Conclusions}

In the case described herein, we demonstrated that the prefabricated KTNME is a useful and simple technique of sparing surgery for the tracheal reconstruction after excessive window resection in a patient with advanced thyroid papillary carcinoma invading the trachea. The offered method of a two-stage sparing treatment leads a long palliative period associated with a good quality of life. Besides, it is economically justified regarding treatment cost minimization. The KTNME applied can adequately restore the frame function of the trachea without postoperative aggravation. As a consequence of the successful application, the KTNME is shown to provide a superior reconstruction and stability for the tracheal grafting during the implantation procedure and thereafter.

\section{Acknowledgment}

This study (research grant No. 8.1.42.2015) was supported by the Tomsk State University Academic D.I. Mendeleev Fund Program in 2015-2016.

\section{Statement of Ethics}

The surgery was performed in accordance with the ethical principles outlined in the Declaration of Helsinki. Its protocol was approved by the Ethical Committee of the Tomsk Cancer Research Institute, and informed consent was obtained from the patient before the upcoming intervention. 


\section{Case Reports in Oncology}

\section{Disclosure Statement}

The authors declare that there are no conflicts of interests regarding the publication of this paper.

\section{References}

1 Gil Z, Patel SG: Surgery for thyroid cancer. Surg Oncol Clin N Am 2008;17:93-120.

2 Ark N, Zemo S, Nolen D, et al: Management of locally invasive well-differentiated thyroid cancer. Surg Oncol Clin N Am 2008;17:145-155.

3 Price DL, Wong RJ, Randolph GW: Invasive thyroid cancer: management of the trachea and esophagus. Otolaryngol Clin North Am 2008;41:1155-1168.

4 Strasser JF, Raben A, Koprowski C: The role of radiation therapy in the management of thyroid cancer. Surg Oncol Clin N Am 2008;17:219-232.

5 Muhamedov M, Kulbakin D, Gunther V, et al: Sparing surgery with the use of TiNi-based endografts in larynx cancer patients. J Surg Oncol 2015;111:231-236.

-6 Aslam R, Steward D: Surgical management of thyroid disease. Otolaryngol Clin North Am 2010;43:273283.

7 Delaere PR, Vranckx JJ, Dooms C, et al: Tracheal autotransplantation: guidelines for optimal functional outcome. Laryngoscope 2011;121:1708-1714.

-8 Javia LR, Zur KB: Laryngotracheal reconstruction with resorbable microplate buttressing. Laryngoscope 2012;122:920-924.

-9 Nomoto Y, Okano W, Imaizumi M, et al: Bioengineered prosthesis with allogenic heterotopic fibroblasts for cricoid regeneration. Laryngoscope 2012;122:805-809.

10 Endo K, Ueno T, Kondo S, et al: Successful treatment of thyroid carcinoma invading the trachea as a single-stage procedure: a case report. Case Rep in Clin Med 2015;4:50-54.

11 Yang CC, Lee H, Wang LS, et al: Resectional treatment for thyroid cancer with tracheal invasion. Arch Surg 2000;135:704-707.

-12 Sandu K, Monnier P: Partial cricotracheal resection with tracheal intussusception and cricoarytenoid joint mobilization: early experience in a new technical variant. Laryngoscope 2011;121:2150-2154.

13 Yener M, Acar GO, Cansiz H, et al: Use of titanium mesh in laryngotracheal reconstruction: an experimental study on rabbits. Eur Arch Otorhinolaryngol 2010;267:1247-1253.

14 Chen W, Ruan Y: Late complications of nickel-titanium alloy stent in tracheal stenosis. Laryngoscope 2012;122:817-820.

15 Nakahira M, Nakatani H, Takeuchi S, et al: Safe reconstruction of a large cervico-mediastinal tracheal defect with a pectoralis major myocutaneous flap and free costal cartilage grafts. Auris Nasus Larynx 2006;33:203-206. 


\section{Case Reports in Oncology}

www.karger.com/cro

Kulbakin et al.: Sparing Surgery for the Successful Treatment of Thyroid Papillary Carcinoma Invading the Trachea: A Case Report
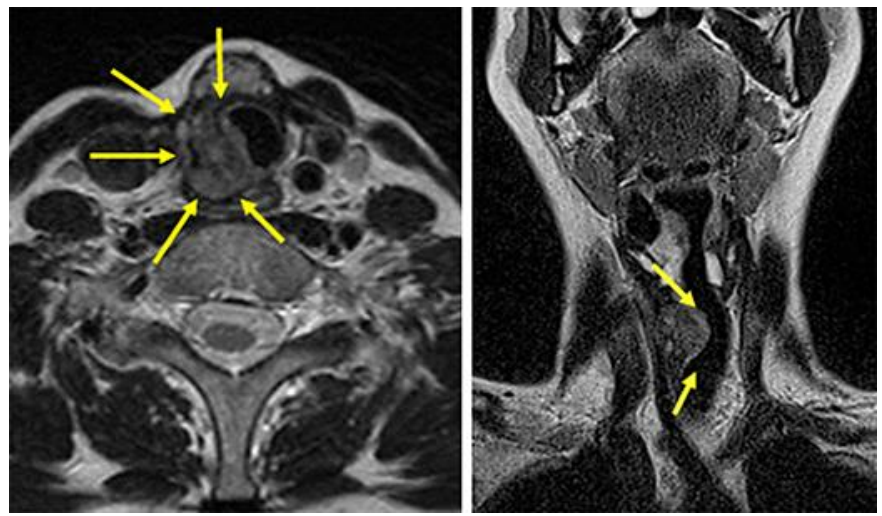

Fig. 1. Cervical MRI showing a thyroid carcinoma invading the tracheal wall and deformed tracheal lumen.
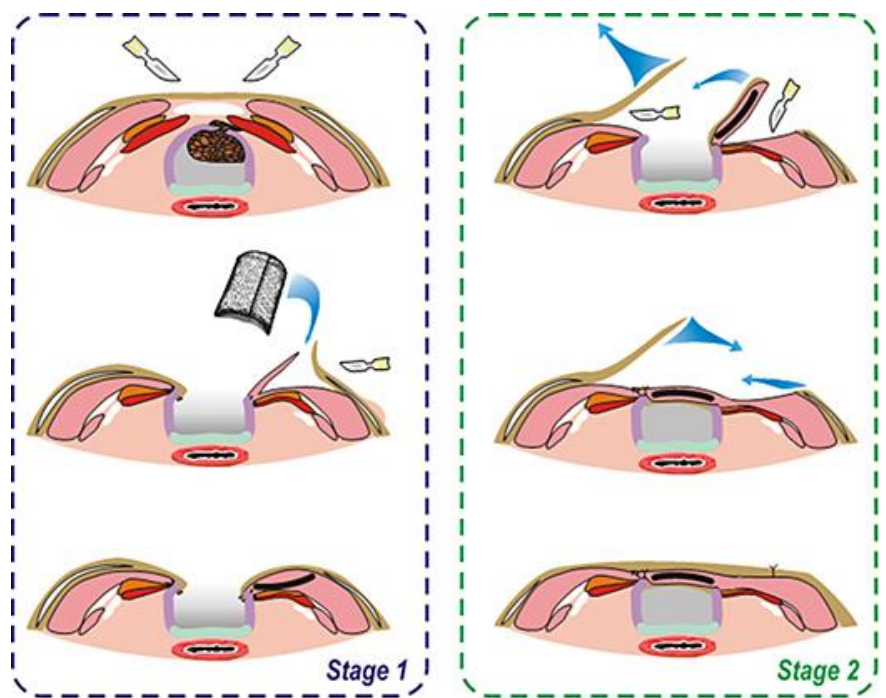

Fig. 2. Outline of the suggested treatment. The defect of the trachea is reconstructed with the prefabricated KTNME and covered with the skin draped over the wound. 


\section{Case Reports in Oncology}

\begin{tabular}{l|l}
\hline Case Rep Oncol 2016;9:772-780 \\
\hline DOI: 10.1159/000452790 & $\begin{array}{l}\text { @ 2016 The Author(s). Published by S. Karger AG, Basel } \\
\text { www.karger.com/cro }\end{array}$ \\
\hline
\end{tabular}

Kulbakin et al.: Sparing Surgery for the Successful Treatment of Thyroid Papillary Carcinoma Invading the Trachea: A Case Report
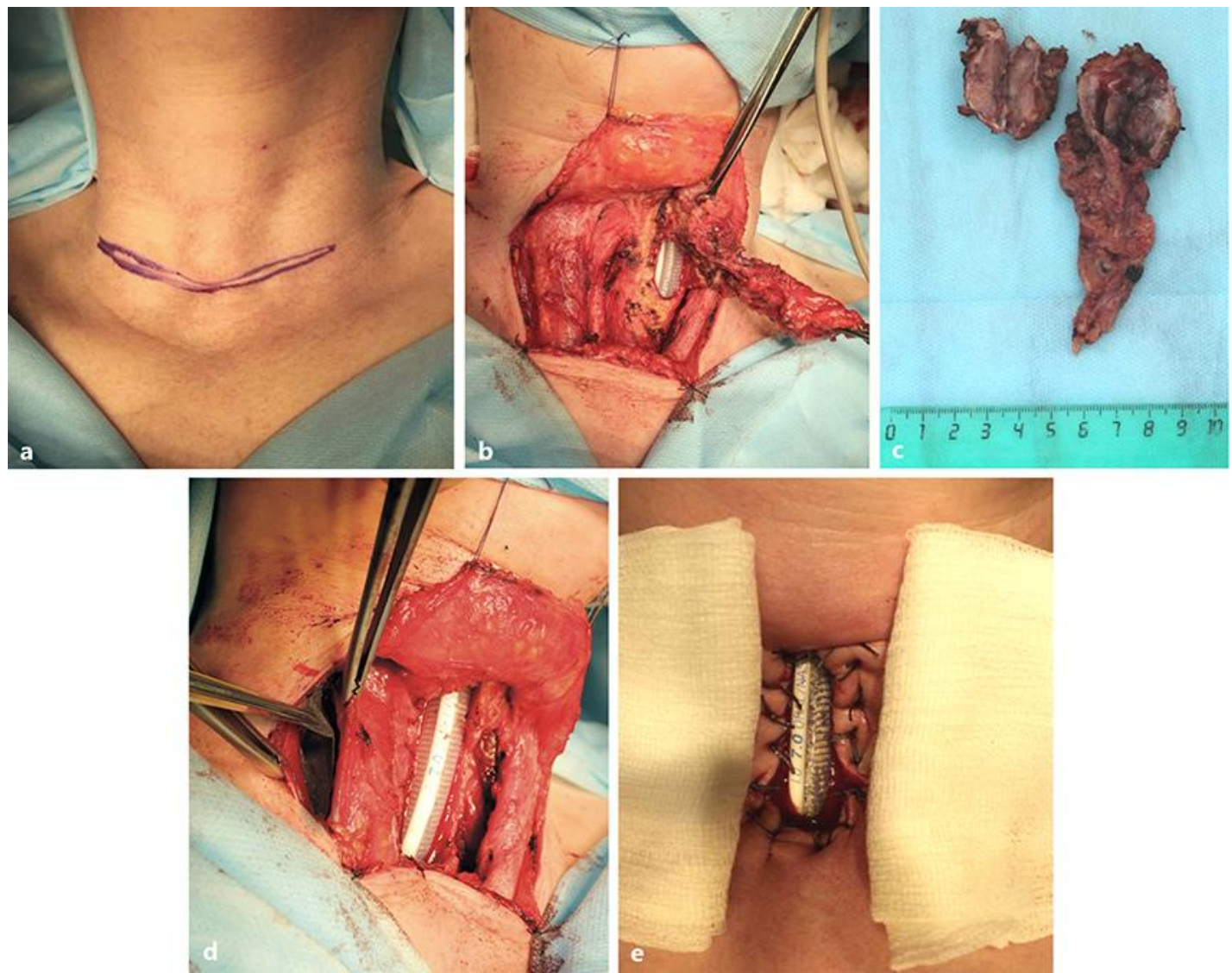

Fig. 3. The first-stage intraoperative findings. a Before U-shape incision. $\mathbf{b}$ The thyroid mass is removed, and a window resection is made. $\mathbf{c}$ The resected tumor specimen is shown. $\mathbf{d}$ The prepared KTNME is implanted into the sternocleidomastoid muscle. e A tracheal window is made with a local skin flap.
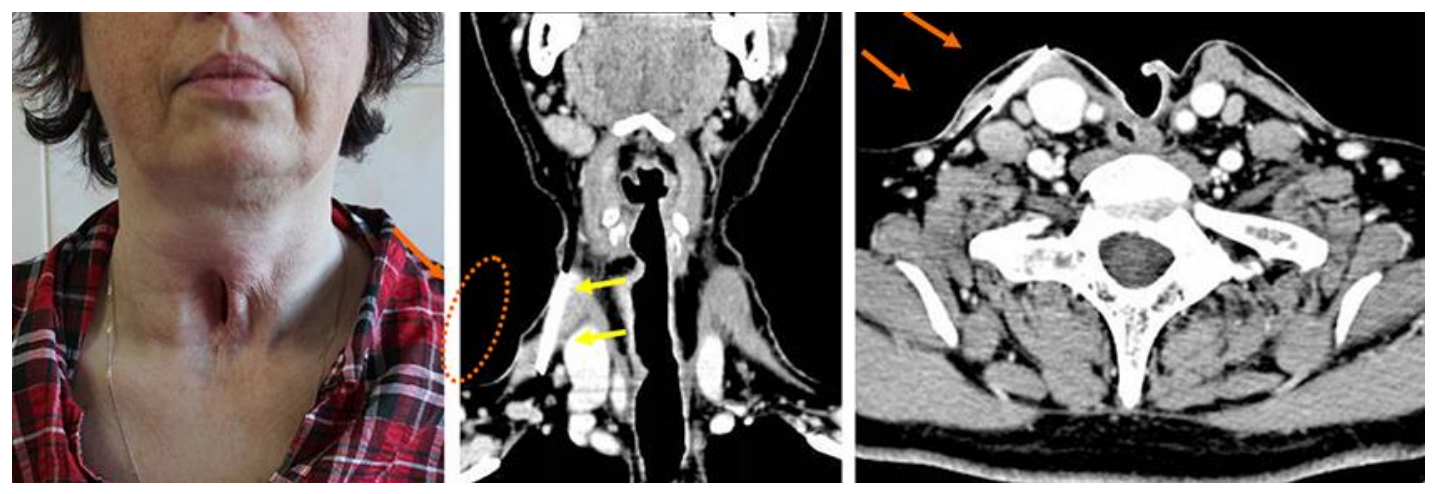

Fig. 4. Three weeks after the tracheal window resection. Postoperative appearance of the patient and cervical MRI showing the implanted KTNME and no stenosis. 


\section{Case Reports in Oncology}

\begin{tabular}{|c|c|}
\hline \multicolumn{2}{|c|}{ Case Rep Oncol 2016;9:772-780 } \\
\hline DOI: $10.1159 / 000452790$ & $\begin{array}{l}\text { (c) } 2016 \text { The Author(s). Published by S. Karger AG, Basel } \\
\text { www.karger.com/cro }\end{array}$ \\
\hline
\end{tabular}
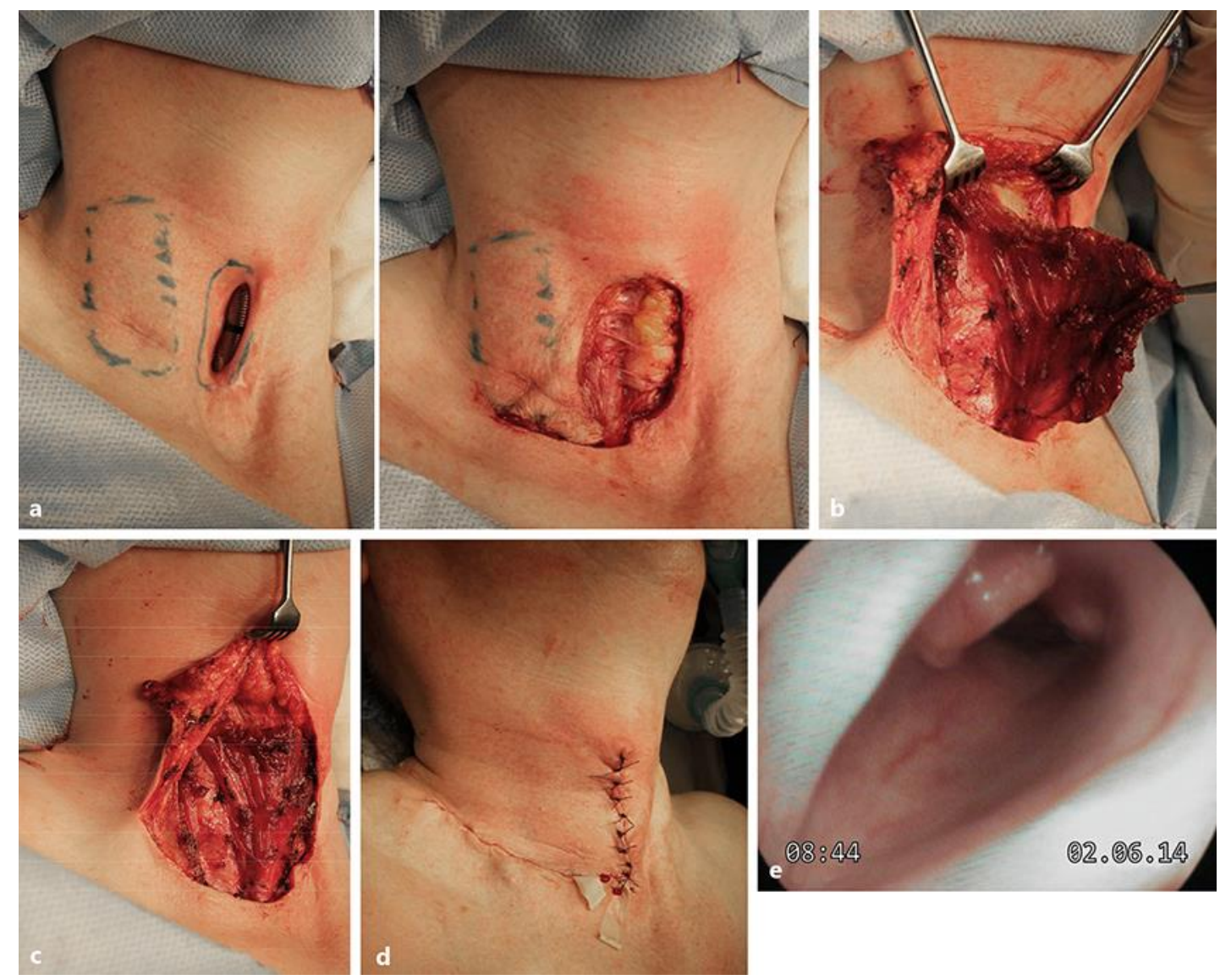

Fig. 5. The second-stage intraoperative findings. a Demarcation and 0-shape incision. The harvested KTNME is separated (b) and overturned to reconstruct the tracheal defect (c). $\mathbf{d}$ The sliding skin flap is swung over the wound. e Postoperative fiberoptic endoscopy before discharge reveals no stenosis or granulation tissue of the lumen, which is well epithelialized. 